\title{
Procyanidins from Vitis vinifera seeds induce apoptotic and autophagic cell death via generation of reactive oxygen species in squamous cell carcinoma cells
}

\author{
YOUNG-SOOL HAH ${ }^{1}$, JIN GU KIM ${ }^{2}$, HEE YOUNG CHO ${ }^{1}$, JIN SUNG PARK ${ }^{3}$, EUN PHIL HEO ${ }^{4}$ and TAE-JIN YOON ${ }^{2}$ \\ ${ }^{1}$ Biomedical Research Institute, Gyeongsang National University Hospital; ${ }^{2}$ Department of Dermatology, \\ Gyeongsang National University Hospital, Institute of Health Sciences, Gyeongsang National University \\ School of Medicine; ${ }^{3}$ Department of Orthopedic Surgery, Gyeongsang National University Hospital, Jinju, \\ Gyeongsangnam-do 52727; ${ }^{4}$ Department of Dermatology, Samsung Changwon Hospital, Sungkyunkwan \\ University School of Medicine, Changwon, Gyeongsangnam-do 51353, Republic of Korea
}

Received July 25, 2015; Accepted September 9, 2016

DOI: $10.3892 / \mathrm{ol} .2017 .6422$

\begin{abstract}
Procyanidins can inhibit cell proliferation and tumorigenesis and induce apoptosis in human skin, breast and prostate carcinoma cell lines. Squamous cell carcinoma (SCC) of the skin is a common form of keratinocytic or non-melanoma skin cancer and is a deadly disease with a poor prognosis due to the ineffectiveness of therapy. The present study aimed to determine whether grape seed proanthocyanidin (GSP) may regulate different modes of cell death in the human SCC12 cell line. The present study found that the treatment of SCC12 cells with GSP inhibited proliferation in a dose-dependent manner and reduced the motility and invasiveness of SCC12 cells through suppression of matrix metalloproteinase-2/9 expression. GSP treatment also resulted in induction of apoptosis and autophagy via generation of reactive oxygen species. The inhibition of autophagy by 3-methyladenine decreased GSP-induced cell death, which suggested that GSP-induced autophagy can promote cell death. The results of the present study suggested that autophagy functions as a death mechanism in SCC and provided a rationale for the use of GSP in combination with autophagy activators for treating cancers such as SCC.
\end{abstract}

\section{Introduction}

Grapes are among the most commonly consumed fruits worldwide. Grape seed extract contains lipids, proteins, carbohydrates and polyphenols. Polyphenols have various biological

Correspondence to: Dr Tae-Jin Yoon, Department of Dermatology, Gyeongsang National University Hospital, Institute of Health Sciences, Gyeongsang National University School of Medicine, 79 Gangnam-ro, Jinju, Gyeongsangnam-do 52727, Republic of Korea E-mail: yoontj@gnu.ac.kr

Key words: procyanidins, squamous cell carcinoma, anticancer activity, apoptosis, autophagy functions and are largely contained in the seeds (60-70\%) and skin (30\%) of grapes (1). Among the phenolic compounds, proanthocyanidins are the dominant fraction in grape seeds and are high molecular weight polymers comprised of dimers or polymers of catechin and epicatechin (2). Grape seed proanthocyanidin (GSP) has a higher antioxidative activity compared with other well-known antioxidants, including vitamin C, vitamin E and gallic acid (3). GSP has been shown to exhibit a wide range of biological activities, including antioxidant, cardioprotective and anti-inflammatory effects (4-6). Furthermore, it was reported that GSP had chemopreventive and antineoplastic effects on breast, prostate, skin and colorectal cancer cells (7-10).

Autophagy is an intracellular degradation system in which proteins and organelles are sequestered, degraded and recycled (11). Autophagy is an important housekeeping process used throughout the body and is involved in the regulation of pathogenesis, including in neurodegenerative and muscular diseases and cancer (12). Under physiological conditions, autophagy controls intracellular homeostasis and is therefore considered a basal cellular mechanism. In addition, autophagy has been described as an alternative route to cell death (termed autophagic or type II programmed cell death) (13) and an adaptation mechanism to numerous physical stresses, including protein aggregation, genotoxic substances and nutrient loss $(14,15)$. Specifically, if a cell's primary mechanism of defense using non-enzymatic molecules, such as flavonoids and vitamins A, C and E, and enzyme-based scavengers, such as catalase, ascorbate peroxidase and superoxide dismutase, fails to achieve the desired outcome under oxidative stress, autophagy acts to remove oxidized materials resulting in protection of the cell (16). However, oxidative stress is not always overcome by these defense mechanisms, and this results in cell death by autophagy (12).

Although apoptotic activation in various type of cancer by GSP has been reported, the precise mechanisms of cancer-associated cell death remain unknown. Therefore, the present study aimed to investigate the effect of GSP on a squamous cell carcinoma (SCC) cell line in order to 
elucidate the potential mechanism of GSP-induced cell death in SCC following GSP treatment. In addition, the role of reactive oxygen species (ROS) in GSP-induced apoptosis and autophagy in SCC was analyzed. Focus was placed on SCC, as it is a lethal disease with a poor prognosis as a result of the ineffectiveness of therapy. The results of the present study indicated a novel function of GSP as an inducer of autophagy and provided evidence for the combined use of autophagy inducers as potentiators of anticancer drugs.

\section{Materials and methods}

Reagents. GSP was provided by Hanlim Pharmaceutical Co., Ltd. (Seoul, Korea). 3-methyladenine (3-MA) was purchased from Sigma-Aldrich (Merck Millipore, Darmstadt, Germany).

Cell culture and treatment. The SCC12 cell line was donated by Dr James Rheinwald (Brigham and Women's Hospital, Harvard Medical School, Boston, USA) and was maintained in growth medium containing a 3:1 ratio of Dulbecco's modified Eagle's medium (DMEM) and Ham's F-12 Nutrient Mixture (Gibco; Thermo Fisher Scientific, Inc., Waltham, MA, USA) supplemented with $10 \%$ fetal bovine serum (FBS), $0.5 \mathrm{mg} / \mathrm{ml}$ hydrocortisone, $5 \mathrm{mg} / \mathrm{ml}$ insulin and $10 \mathrm{ng} / \mathrm{ml}$ epidermal growth factor. The cells were treated with various concentrations of GSP dissolved in PBS. Control cells received the same volume of the vehicle solution (PBS).

Cell viability analysis. SCC12 cells $\left(5 \times 10^{4}\right.$ cells/well) were seeded into 24 -well plates and incubated at $37^{\circ} \mathrm{C}$ for $24 \mathrm{~h}$, after which the cells were treated with increasing concentrations of GSP $(10,50,100$ and $200 \mu \mathrm{g} / \mathrm{ml})$ for $24 \mathrm{~h}$. The cell viability was assessed using Cell Counting kit-8 (CCK-8) assays (Dojindo Molecular Technologies, Inc., Kumamoto, Japan). Briefly, CCK-8 solution $(10 \mu \mathrm{l})$ was added to each well and incubated for $1 \mathrm{~h}$ at $37^{\circ} \mathrm{C}$ in a humidified atmosphere containing $5 \% \mathrm{CO}_{2}$. Absorbance was then measured at $450 \mathrm{~nm}$ using a microplate reader (Molecular Devices, LLC, Sunnyvale, CA, USA). The effect of 3-MA on cell death was determined after the cells had been treated for $24 \mathrm{~h}$. Cells were pre-incubated with 3-MA (10 mM) for $1 \mathrm{~h}$ prior to the addition of GSP.

Flow cytometric DNA analysis. The collected cells were washed twice with cold PBS, fixed with $70 \%$ ethanol for $1 \mathrm{~h}$ at $4^{\circ} \mathrm{C}$, treated with $1 \mathrm{mg} / \mathrm{ml}$ RNase A (Sigma-Aldrich; Merck Millipore) and then stained with $50 \mu \mathrm{g} / \mathrm{ml}$ propidium iodide (Sigma-Aldrich; Merck Millipore). The relative DNA content per cell was determined using a FACSCalibur ${ }^{\mathrm{TM}}$ flow cytometer (BD Biosciences, Franklin Lakes, NJ, USA). Data were analyzed with CellQuest Pro software (version 4.0; BD Biosciences). The percentage of cells in each phase of the cell cycle was calculated using ModFit LT Software (version 3.0; Verity Software House, Inc., Topsham, ME, USA).

Cell migration assay. SCC12 cells were seeded at a density of $2 \times 10^{5}$ cells/well onto 24 -well plates and incubated at $37^{\circ} \mathrm{C}$ overnight. Subsequent to the cell monolayer being formed, horizontal lines were scraped onto the bottom of each well using a 200- $\mu$ l yellow pipette tip. The cells were then washed with PBS and incubated for $6 \mathrm{~h}$ with serum-free medium containing 10 or $50 \mu \mathrm{g} / \mathrm{ml}$ GSP. Images of the plates were captured using an inverted microscope equipped with an image capture system (Nikon Corporation, Tokyo, Japan).

Invasion assay. Invasion assays were performed on 24-well Transwell inserts (Costar; Sigma-Aldrich; Merck Millipore) with polycarbonate filters $(8-\mu \mathrm{m}$ pore size). The Transwell inserts were coated with a uniform layer of BD Matrige ${ }^{\mathrm{TM}}$ Basement Membrane Matrix (BD Biosciences). Stable cell lines were resuspended in DMEM/F12 containing $10 \%$ FBS and seeded into the upper wells $\left(1 \times 10^{5}\right.$ cells/well $)$ and incubated at $37^{\circ} \mathrm{C}$ for $24 \mathrm{~h}$. Invaded cells were fixed in $4 \%$ paraformaldehyde, stained with DAPI and counted under a fluorescent microscope at x100 magnification for 5 random fields.

Gelatin zymography. The net gelatinase [matrix metalloproteinase (MMP)-2 and -9] activity of cell culture media supernatants was determined using SDS-containing gels prepared by copolymerizing acrylamide and gelatin at a final concentration of $0.1 \%(\mathrm{w} / \mathrm{v})$. Samples were dispersed for $10 \mathrm{~min}$ at room temperature in Laemmli solubilizing solution from which dithiothreitol had been omitted. Electrophoresis was performed at $10 \mathrm{~mA}$ and $4^{\circ} \mathrm{C}$ for $3 \mathrm{~h}$. Subsequent to electrophoresis, the gels were incubated for $60 \mathrm{~min}$ at room temperature in the zymogram renaturing buffer containing $2.5 \%$ (v/v) Triton X-100, followed by $16 \mathrm{~h}$ incubation at $37^{\circ} \mathrm{C}$ in $50 \mathrm{mM}$ Tris- $\mathrm{HCl}(\mathrm{pH} 7.6)$ containing $5 \mathrm{mM} \mathrm{CaCl}_{2}$. The gels were stained with $0.5 \%$ Coomassie Brilliant Blue G-250, according to a standard protocol.

Western blot analysis. The cells were lysed by incubation for 30 min with NP-40 lysis buffer (20 mM Tris, pH 7.5, $140 \mathrm{mM}$ $\mathrm{NaCl}, 1 \mathrm{mM}$ EDTA) containing $1 \%$ (v/v) Nonidet P-40, $5 \mu \mathrm{M}$ AEBSF, $1.5 \mathrm{nM}$ aprotinin, $10 \mathrm{nM}$ E-64 and $10 \mathrm{nM}$ Leupeptin. Cells were sonicated and centrifuged at $12,000 \mathrm{x}$ g for $10 \mathrm{~min}$ at $4^{\circ} \mathrm{C}$ to remove insoluble debris. Total proteins $(30 \mu \mathrm{g})$ were separated by $10 \%$ SDS-PAGE and transferred onto a nitrocellulose membrane using semidry transfer apparatus (Trans-Blot SD Semi-Dry Transfer Cell; Bio-Rad Laboratories, Inc., Hercules, CA, USA) at $15 \mathrm{~V}$ for $30 \mathrm{~min}$. Membranes were blocked with $5 \%$ skimmed milk and incubated with specific antibodies against MMP-2 (1:1,000 dilution; sc-10736; Santa Cruz Biotechnology, Inc., Dallas, TX, USA) and microtubule-associated protein 1 light chain 3 (LC3) (1:1,000 dilution; AP1801a; Abgent, Inc., San Diego, CA, USA) at $4^{\circ} \mathrm{C}$ overnight. After three washes with Tris-buffered saline containing $0.1 \%$ Tween-20, membranes were incubated with horseradish peroxidase-conjugated secondary antibody (1:3,000 dilution; sc-2030; Santa Cruz Biotechnology, Inc.). Protein bands were identified by the enhanced chemiluminescence detecting system (Pierce; Thermo Fisher Scientific Inc.), according to the manufacturer's protocol. $\beta$-actin was used as a loading control in the stripped blot.

ROS measurement. Intracellular generation of ROS was determined using 2',7'-dichlorodihydrofluorescein diacetate (DCF-DA; Molecular Probes, Thermo Fisher Scientific Inc.). The dye that integrated into the cells was deacetylated by intracellular esterases. Upon oxidation, DCF-DA is converted 
to highly fluorescent 2,7-dichlorofluorescein (17). Briefly, cells were cultured at $37^{\circ} \mathrm{C}$ overnight in 6-well plates and then treated with GSP in the presence or absence of $\mathrm{N}$-acetyl cysteine (NAC) for $4 \mathrm{~h}$. The cells were stained with $5 \mu \mathrm{M}$ DCF-DA in serum-free medium for $15 \mathrm{~min}$ and removed from the plate with trypsin-EDTA (Gibco, Thermo Fisher Scientific, Inc.). The fluorescence intensity of the cells was determined by flow cytometry with an excitation wavelength of $480 \mathrm{~nm}$ and an emission wavelength of $525 \mathrm{~nm}$ (BD Biosciences). Data were analyzed using CellQuest Pro software (version 4.0, BD Biosciences).

Recombinant adenovirus. An adenovirus encoding a green fluorescent protein (GFP)-tagged LC3 (Ad-GFP-LC3) was created using the Virapower adenovirus expression system (Invitrogen; Thermo Fisher Scientific, Inc.), according to the manufacturer's protocol. Briefly, a DNA construct encoding GFP fused to LC3 was subcloned into the pENTR vector. Site-specific recombination between entry vectors (pENTR-GFP-LC3) and the adenoviral destination vector (pAd/CMV/V5-DEST) was performed with LR clonase II (Invitrogen; Thermo Fisher Scientific, Inc.). All constructs were verified by performing Sanger dideoxy sequencing analysis (Bioneer Inc., Seoul, Korea). The verified clone (pAd-GFP-LC3) was linearized using Pac I (New England Biolabs, Inc., Ipswich, MA, USA) and then transfected into 293A cells with Lipofectamine 2000 (Invitrogen; Thermo Fisher Scientific, Inc.). Subsequent to amplification, viruses from the culture supernatants of 293 cells that showed cytopathogenic effects were purified using the Adeno- $\mathrm{X}^{\mathrm{TM}}$ Virus Purification kit (BD Biosciences) and viral titers were determined using a plaque-forming assay with serial dilutions. Briefly, the cells were plated onto 6-well plates at a density of $1 \times 10^{5}$ cells $/ \mathrm{ml}$ and infected with recombinant adenoviruses at a multiplicity of infection of 10 on the following day.

Detection of autophagy. Cells were infected with Ad-GFP-LC3 adenovirus and treated with different concentrations of GSP for $12 \mathrm{~h}$. GFP-LC3 protein puncta-formation in the cells was observed under a confocal microscope (Olympus FV-1000; Olympus Corporation, Tokyo, Japan).

Statistical analysis. Data were analyzed using a two-tailed Student's t-test. Statistical analyses were performed using SPSS software, version 7.0 (SPSS Inc., Chicago, IL, USA). $\mathrm{P}<0.05$ was considered to indicate a statistically significant difference.

\section{Results}

GSP induces cell cycle arrest and apoptosis in SCC12 cells. The effect of GSP on cell viability in human SCC12 cells was examined using CCK-8 assays. GSP caused a dose-dependent reduction of SCC12 cell viability, and treatment with $100 \mu \mathrm{g} / \mathrm{ml}$ GSP for $24 \mathrm{~h}$ resulted in an $\sim 50 \%$ reduction in cell viability $(\mathrm{P}<0.01$; Fig. 1). Flow cytometry was used to investigate whether the reduction of cell viability by GSP was due to apoptosis. Apoptosis was evaluated by the measurement of the number of cells in the sub-G1 region. As shown in Fig. $2 \mathrm{~A}$ and B, GSP induced a significant and dose-dependent increase in the percentage of cells in the sub-G1 region $(\mathrm{P}<0.05$ at $50 \mu \mathrm{g} / \mathrm{ml}$

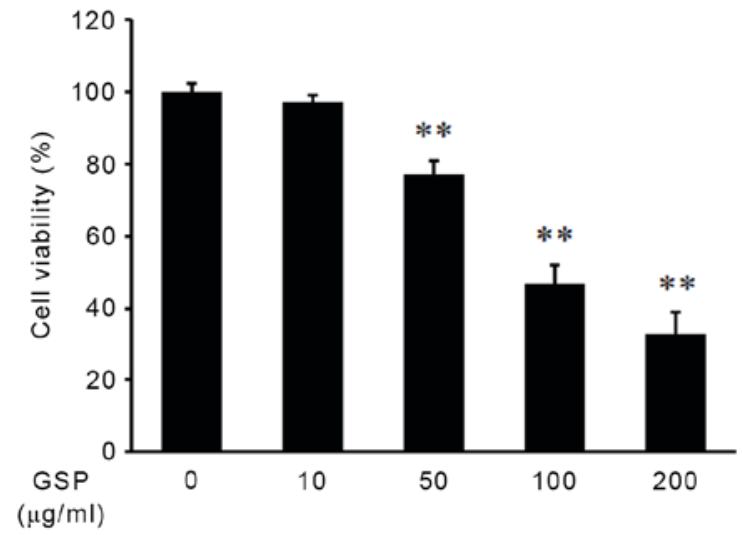

Figure 1. GSP suppresses cell proliferation/viability in a dose-dependent manner. Cells were treated with increasing concentrations of GSP then incubated at $37^{\circ} \mathrm{C}$ for $24 \mathrm{~h}$. The cell viability was determined using Cell Counting kit- 8 assays. The cell viability was expressed as a percentage of the untreated cells. Data are presented as the mean \pm standard deviation of at least three independent experiments. ${ }^{* *} \mathrm{P}<0.01 \mathrm{vs} .0 \mu \mathrm{g} / \mathrm{ml}$ GSP. GSP, grape seed procyanidins.

GSP, $\mathrm{P}<0.01$ at 100 and $200 \mu \mathrm{g} / \mathrm{ml}$ GSP). These data indicate that the anti-proliferative effect of GSP on SCC12 cells may be associated with the induction of apoptosis.

The present study next investigated the possible effect of GSP on cell cycle progression. GSP treatment resulted in an increase in the number of cells in the $G_{2} / M$ phase of the cell cycle, while the number of cells in the $G_{0} / G_{1}$ phase was decreased compared with the control $(\mathrm{P}<0.05$ at $100 \mu \mathrm{g} / \mathrm{ml}$ GSP, $\mathrm{P}<0.01$ at $200 \mu \mathrm{g} / \mathrm{ml}$ GSP; Fig. 2C). This result indicates that GSP treatment induces the $\mathrm{G}_{2} / \mathrm{M}$ arrest in SCC12 cells.

GSP inhibits the motility and invasiveness of SCC12 cells through suppression of MMP-2/9 expression. SCC12 cells have previously been reported to have a high metastatic potential and GSP has been shown to inhibit the migration of cancer cells by disrupting the mitochondrial pathway and increasing the activation of caspase-3 (18). However, the mechanism underlying the inhibitory effect of GSP on the motility of SCC cells has yet to be investigated. Therefore, the effect of GSP on the motility and invasive abilities of SCC12 cells was analyzed in the present study. Cell motility was investigated with a wound-healing assay. GSP-treated SCC12 cells showed a marked reduction of cell motility compared with the control (Fig. 3A). Using the Matrigel invasion assay, a decrease in cell invasion was observed in GSP-treated SCC12 cells, as compared with the control (Fig. 3B). Subsequently, the effect of GSP on the activities of MMP-2 and -9 was evaluated using a gelatin zymogram analysis, and it was shown that GSP inhibited the activities of MMP-2 and -9 in SCC12 cells in a dose-dependent manner (Fig. 3C-a). Since the inhibition of MMP-2 and -9 activities is associated with the expression level of MMP-2 and -9, the present study evaluated the expression levels of MMP-2. GSP downregulated the protein expression of MMP-2 in SCC12 cells (Fig. 3C-b).

GSP induced-cytotoxicity of SCC12 cells involves autophagic cell death and apoptosis. To assess the effect of GSP on autophagy induction, the present study performed two 
A
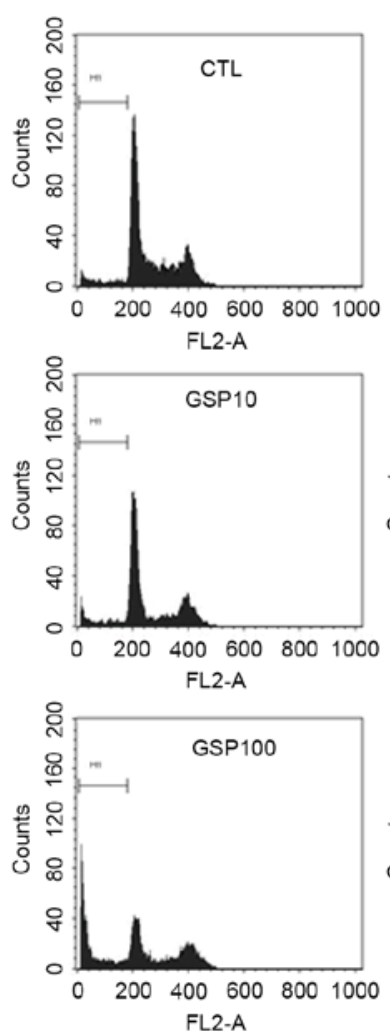
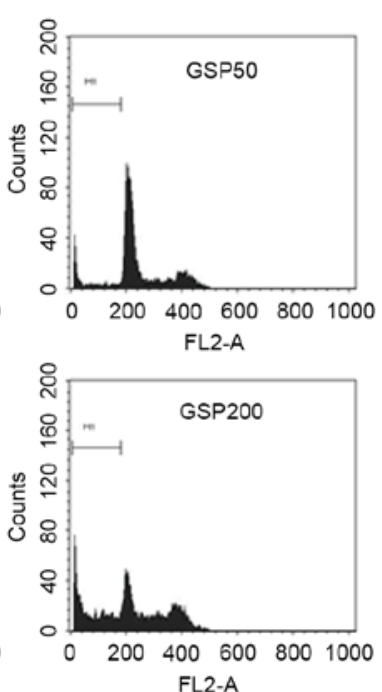

B

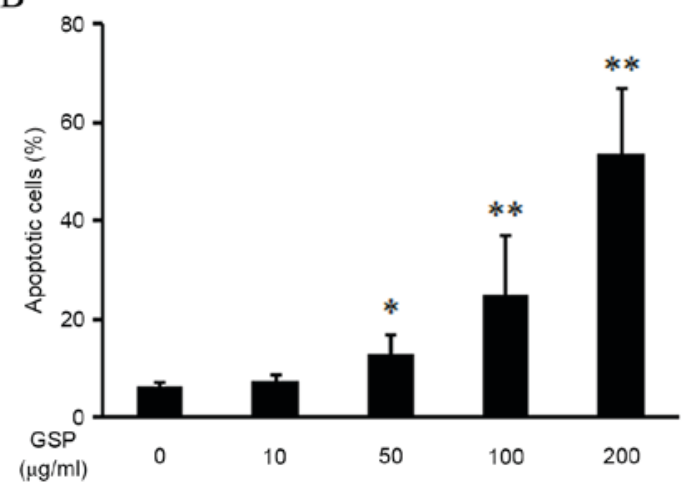

$\mathrm{C}$

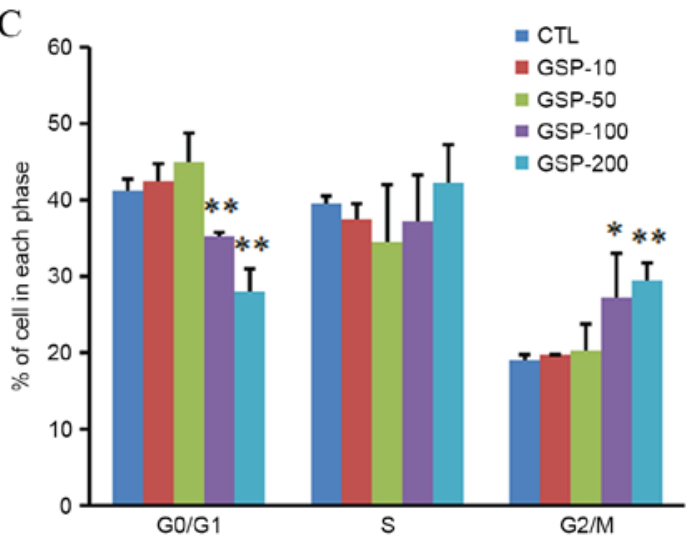

Figure 2. GSP induces apoptosis and changes in the cell cycle distribution. (A) Representative images of flow cytometry analysis. The cell cycle distribution was determined by propidium iodide staining and flow cytometry. (B) Statistical plots of flow cytometry analysis for apoptotic cells and (C) histograms of cell cycle alterations. Data are presented as the mean \pm standard deviation of three independent experiments. ${ }^{*} \mathrm{P}<0.05,{ }^{* *} \mathrm{P}<0.01$ vs. the CTL. GSP, grape seed procyanidins; CTL, control.

A
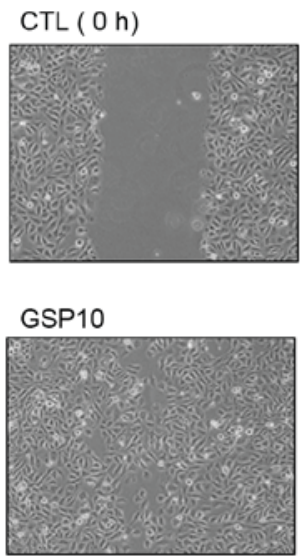

GSP100

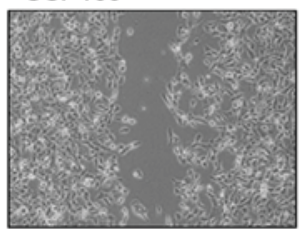

CTL $(6 \mathrm{~h})$

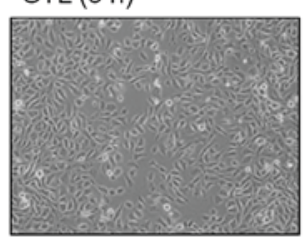

GSP50

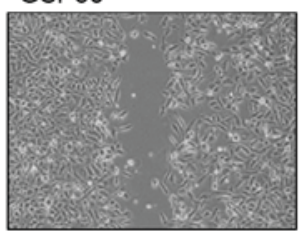

GSP200

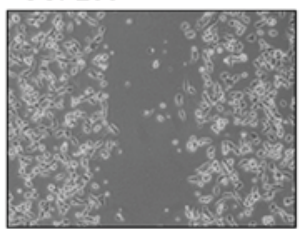

B

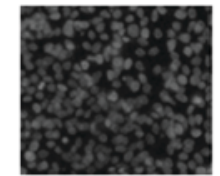

0

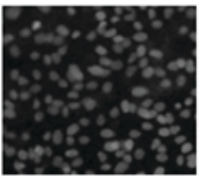

10

$\operatorname{GSP}(\mu \mathrm{g} / \mathrm{ml})$

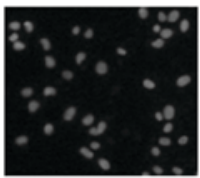

50
$\mathrm{Ca}$

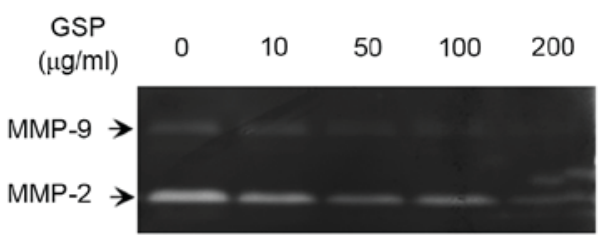

b

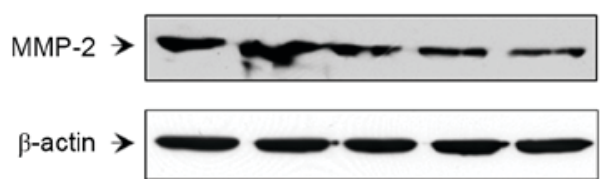

Figure 3. GSP inhibits the migration and invasion of SCC12 cells by reducing MMP-2/9 expression and activity. (A) The effect of GSP on the motility of SCC12 cells was assessed using wound-healing assays. Images were captured of migrating cells in the denuded zone (magnification, x100). (B) The effect of GSP on the invasion of SCC12 cells. Cells were treated with various concentrations of GSP for $24 \mathrm{~h}$ and a cell invasion assay was performed. Images were captured of the invaded cells (magnification, x200). (C) The effect of GSP on the activities and protein expression of MMP-2/9. (C-a) Supernatants of GSP-treated SCC12 cells were loaded into $10 \%$ gelatin-containing gel, resolved by electrophoresis and stained with Coomassie blue. (C-b) Total cell lysates were analyzed by SDS-PAGE, followed by probing with anti-human MMP-2 antibody. $\beta$-actin was used as a control for protein loading. MMP, matrix metalloproteinase; GSP, grape seed procyanidins; CTL, control. 
A

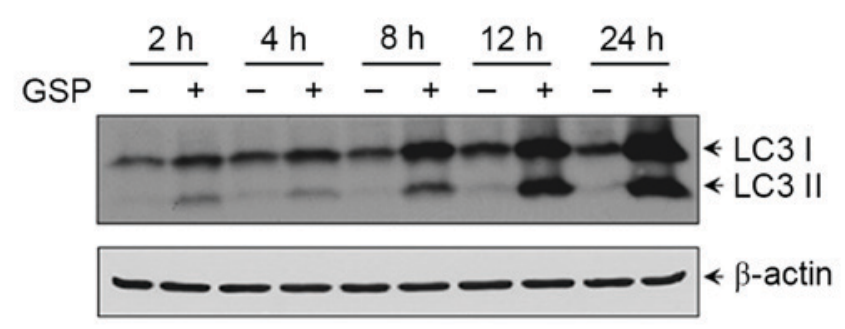

B

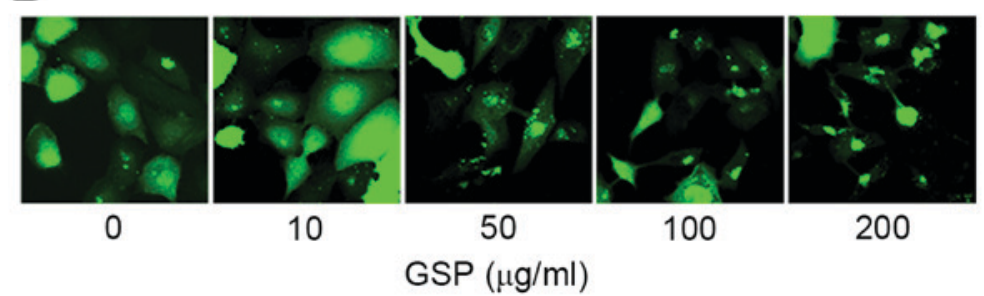

Figure 4. GSP induces autophagy in SCC12 cells. (A) Cells were treated with vehicle control (PBS) or GSP (100 $\mu \mathrm{g} / \mathrm{ml})$ for the indicated time period prior to being analyzed for LC3-II formation by immunoblotting with an anti-LC3 antibody. $\beta$-actin was used as a control for protein loading. (B) Cells were infected with Ad-GFP-LC3 overnight and then treated with the indicated concentrations of GSP. Subsequent to $12 \mathrm{~h}$ of treatment, the fluorescence of GFP-LC3 was observed under a confocal microscope. GSP, grape seed procyanidins; LC3, microtubule-associated protein 1 light chain 3; Ad, adenovirus; GFP, green fluorescent protein.

independent LC3 analyses: LC3-II production and formation of GFP-LC3 puncta. Upon induction of autophagy, LC3 is lipidated and aggregates onto the membranes of autophagic vacuoles (19). Following treatment with $100 \mu \mathrm{g} / \mathrm{ml} \mathrm{GSP}$, the protein expression of LC3-I and LC3-II was increased in a time-dependent manner (Fig. 4A). To further confirm GSP-induced autophagy, a recombinant adenovirus expressing the GFP-LC3 fusion protein was created and whether GSP was able to promote GFP-LC3 puncta formation, an indicator of autophagosome generation (20), was investigated. Ad-GFP-LC3-infected SCC12 cells were treated with GSP and the GFP-LC3 fusion protein was visualized under a confocal microscope. GFP-LC3 was observed to form punctate structures and the number of GFP-LC3 puncta was increased in a dose-dependent manner (Fig. 4B). The GFP-LC3 exhibited a diffuse nuclear and cytosolic distribution in control cells.

Autophagy has also been implicated in a type of programmed cell death type II termed autophagic cell death (13). The present study next examined whether, besides apoptosis, GSP was able to induce autophagic cell death. Cell viability assays were conducted using cells exposed to GSP in the presence or absence of 3-methyladenine (3-MA), an autophagy inhibitor. Pre-treatment of the cells with 3-MA significantly reduced GSP-induced cytotoxicity (GSP vs. $3-\mathrm{MA}+\mathrm{GSP}, \mathrm{P}<0.05$; Fig. 5A), but failed to block GSP-induced apoptosis (Fig. 5B). These results suggest that the anti-proliferative activity of GSP may result, at least in part, from autophagy-mediated cell death.

ROS modulation is involved in GSP-induced apoptosis and autophagy. The intracellular ROS level was determined using the ROS-sensitive fluorescent dye DCF-DA. ROS accumulation increased in a dose-dependent manner in GSP-treated SCC1 2 cells (Fig. 6A). However, GSP-induced ROS generation was significantly blocked by pretreatment of the cells with the antioxidant agent, NAC (Fig. 6B).
To determine whether elevated ROS levels mediate the cytotoxic effects of GSP, the effects of antioxidant agents on cell viability was examined. Cells were pretreated with $10 \mathrm{mM}$ NAC and then treated with $100 \mu \mathrm{g} / \mathrm{ml} \mathrm{GSP}$ for an additional $24 \mathrm{~h}$. As shown in Fig. 7A, GSP-induced cell death was significantly prevented by pretreatment with NAC (GSP vs. NAC+GSP, $\mathrm{P}<0.01$ ). These results suggest that GSP-induced apoptosis and autophagy may be associated with its activity in enhancing the intracellular levels of ROS. To investigate this possibility, it was first determined whether preincubation with NAC was able to inhibit GSP-induced apoptosis. Notably, pretreatment with NAC significantly suppressed GSP-induced apoptosis of SCC12 cells (GSP vs. NAC+GSP, P<0.01; Fig. 7B). To further analyze the involvement of ROS in autophagy, the effect of GSP on autophagic activation in the presence of NAC was assessed. GFP-LC3 puncta formation by GSP was markedly impeded in the presence of NAC (Fig. 7C). These observations suggest that GSP-induced oxidative stress may activate apoptosis and autophagy pathways in SCC12 cells. Based on the results obtained, the present study has proposed a mechanism for GPS-induced cell death (Fig. 8) that involved ROS-mediated activation of autophagy and apoptosis.

\section{Discussion}

The present study aimed to investigate the effects of GSP on SCC1 2 cells, and various responses were detected. The proliferation of SCC12 cells was systemically inhibited by GSP in a dose-dependent manner. In previous studies, apoptosis was thought to be the cause of the GSP-mediated inhibition of cancer cell proliferation $(7-10,18)$. These anti-proliferative effects may be attributed to alterations in various intracellular mechanisms, including suppression of cellular proliferation, growth arrest at cell cycle checkpoints and enhanced apoptosis induction (21). Numerous cancer cells have been shown to 
A

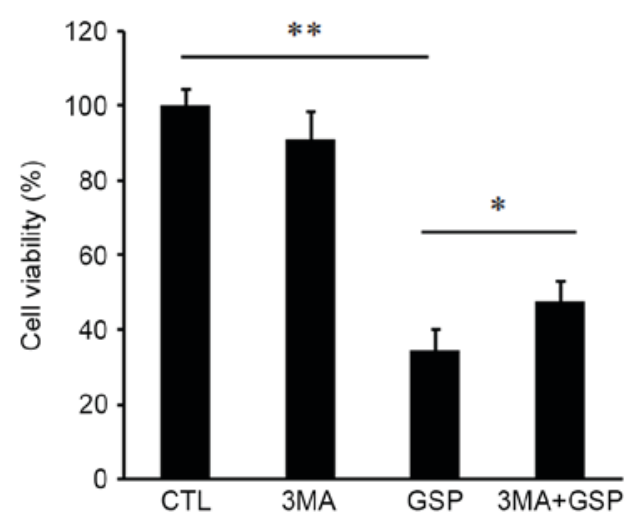

B

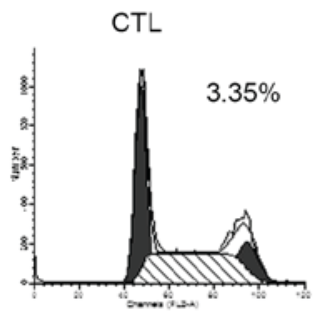

GSP

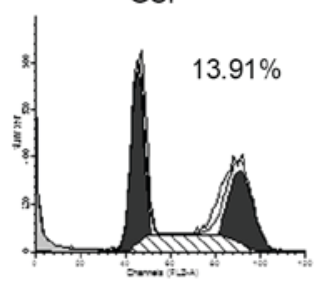

3MA

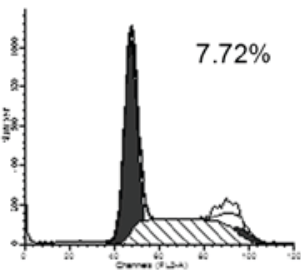

$3 M A+G S P$

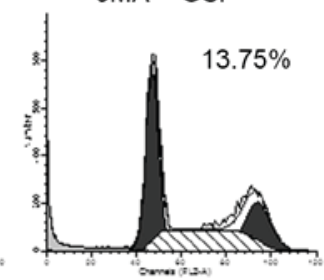

Figure 5. 3-MA, an autophagy inhibitor, diminishes GSP-induced cytotoxicity. (A) Cells were treated with GSP (100 $\mu \mathrm{g} / \mathrm{ml})$ with or without 3-MA (10 mM). Cell viability was determined by Cell Counting kit-8 assays. Data are presented as the mean \pm standard deviation of at least three independent experiments. ${ }^{*} \mathrm{P}<0.05,{ }^{* *} \mathrm{P}<0.01$. (B) SCC12 cells were exposed to $50 \mu \mathrm{g} / \mathrm{ml}$ GSP. Apoptotic cells (sub-G1) were determined after $24 \mathrm{~h}$ of treatment by propidium iodide staining and FACS. Original FACS plots are presented and the mean values of three independent experiments are shown. 3-MA, 3-methyladenine; GSP, grape seed procyanidins; FACS, fluorescence-activated cell sorting.

A

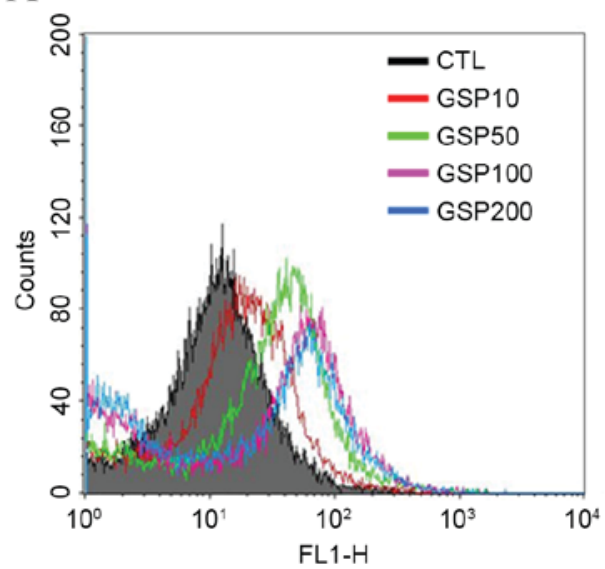

$\mathrm{B}$

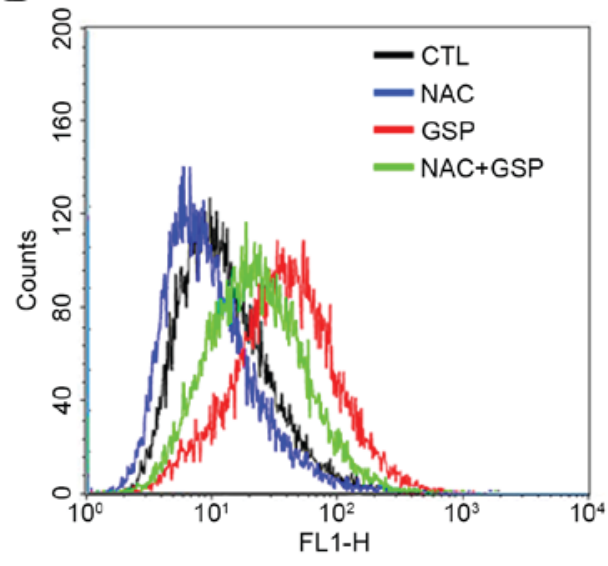

Figure 6. GSP induces ROS production in SCC12 cells. (A) Cells were treated with vehicle control or GSP (indicated doses) for $6 \mathrm{~h}$. To determine intracellular ROS levels, the cells were incubated for $15 \mathrm{~min}$ with the ROS-sensitive fluorophore DCF-DA $(5 \mu \mathrm{M})$. ROS production was determined by fluorescence-activated cell sorting analysis. (B) Cells were treated with GSP $(50 \mu \mathrm{g} / \mathrm{ml})$ in the presence or absence of NAC $(10 \mathrm{mM})$ for $6 \mathrm{~h}$, followed by incubation with DCF-DA $(5 \mu \mathrm{M})$. Cells were examined by flow cytometry. GSP, grape seed procyanidins; ROS, reactive oxygen species; DCF-DA, 2',7'-dichlorodihydrofluorescein diacetate; NAC, $\mathrm{N}$-acetyl cysteine.

have defects in the cell cycle, which allows them to proliferate uncontrollably. Conversely, in normal cells, cell cycle progression is regulated by cell cycle check points (22). The majority of the chemopreventive agents induce either $G_{1} / S$ phase or $G_{2} / M$ phase arrest, which prevents uncontrolled cell division $(23,24)$. In the present study, the cell growth inhibition induced by GSP was associated with a moderate accumulation in the $G_{2} / M$ phase of the cell cycle, with a corresponding decrease in the percentage of $\mathrm{G}_{1}$ phase cells. Additionally, a noticeable sub- $\mathrm{G}_{1}$ apoptotic population was evident in the histogram of SCC12 cells treated with various concentrations of GSP, and the size of this population increased in a dose-dependent manner.

To the best of our knowledge, the present study is the first to report a novel function of GSP: The induction of autophagy, as shown by activation of the autophagosomal marker LC3.
Autophagy may have proapoptotic or antiapoptotic functions depending on the cell type and stimulus (25). The present study provides evidence to show the induction of autophagic and apoptotic machineries in SCC12 cells upon treatment with GSP, suggesting that GSP induces cell death by apoptosis and autophagy. These results were not unexpected, as numerous natural products are known to possess antioxidant and anticancer properties (26-28). In a previous study, GSP inhibited oxidative stress-induced apoptosis by elevating the cellular antioxidant capacity (29). Conversely, other studies reported that GSP exerted antiproliferative activity by inducing the apoptosis of cancer cells $(7-10,18)$. Despite the antioxidant action of GSP, the present study demonstrated that GSP exhibited pro-oxidant activity resulting in selective cell death of SCC1 2 cells. 
A

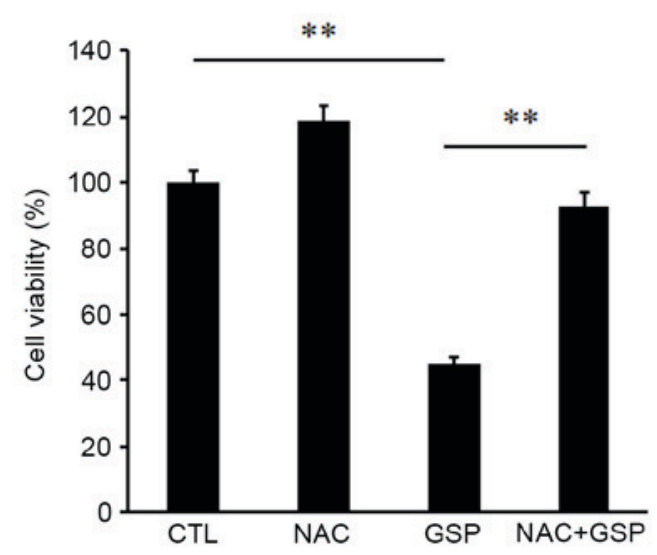

C

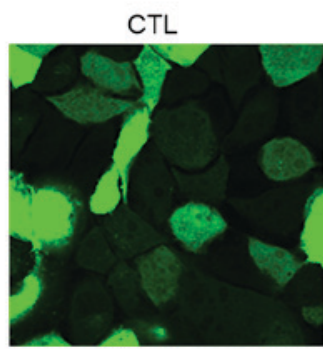

B

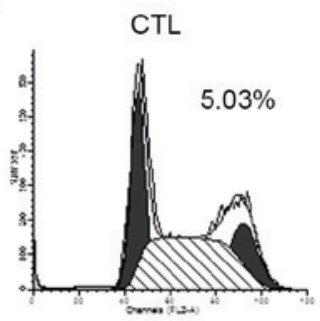

GSP
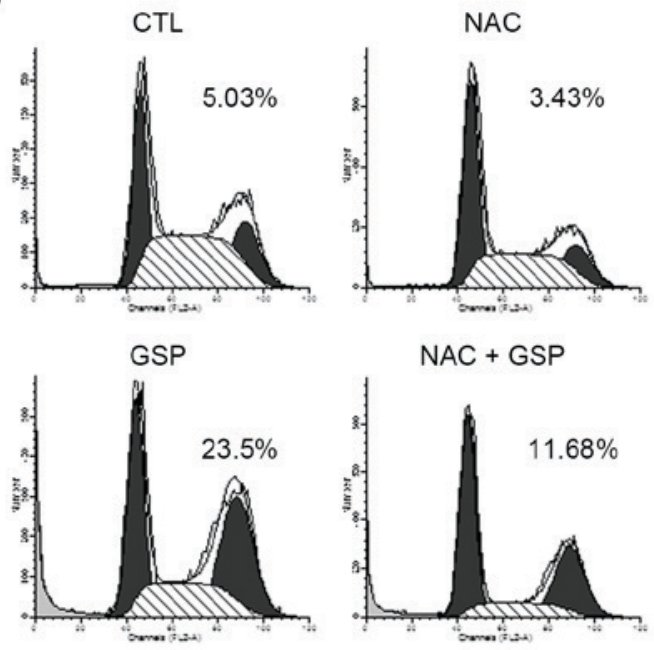

$N A C+G S P$

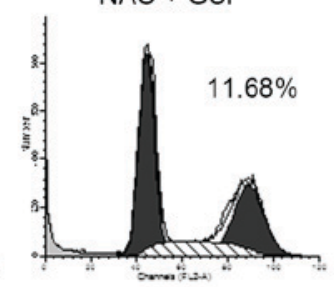

GSP
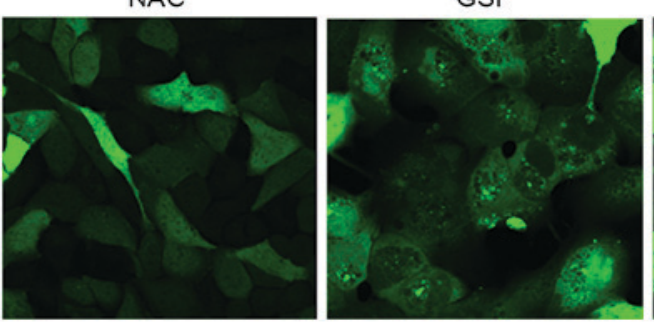

$\mathrm{NAC}+\mathrm{GSP}$

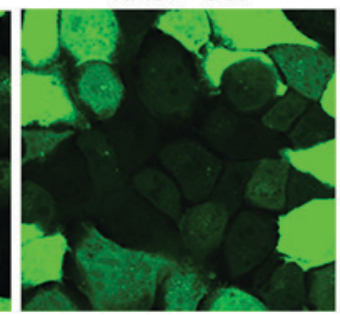

Figure 7. Scavenging of ROS inhibits apoptosis and autophagy. (A) SCC12 cells were pretreated with $10 \mathrm{mM} \mathrm{NAC}$, followed by co-treatment with $100 \mu \mathrm{g} / \mathrm{ml}$ GSP for an additional $24 \mathrm{~h}$. Cell viability was determined by Cell Counting kit-8 assays. Data are presented as the mean \pm standard deviation of at least three independent experiments. " $\mathrm{P}<0.01$. (B) SCC12 cells were treated as in (A) and sub-G1 GSP-treated cells were determined by flow cytometry. Original FACS plots are presented and the mean values of three independent experiments are shown. (C) Ad-GFP-LC3-infected SCC12 cells were pre-incubated with $10 \mathrm{mM}$ NAC for $1 \mathrm{~h}$ prior to $6 \mathrm{~h}$ incubation with $100 \mu \mathrm{g} / \mathrm{ml} \mathrm{GSP}$. Cells were fixed and examined under a confocal microscope (magnification, x200). ROS, reactive oxygen species; NAC, N-acetyl cysteine; GSP, grape seed procyanidins; FACS, fluorescence-activated cell sorting; Ad, adenovirus; GFP, green fluorescent protein; LC3, microtubule-associated protein 1 light chain 3; CTL, control.

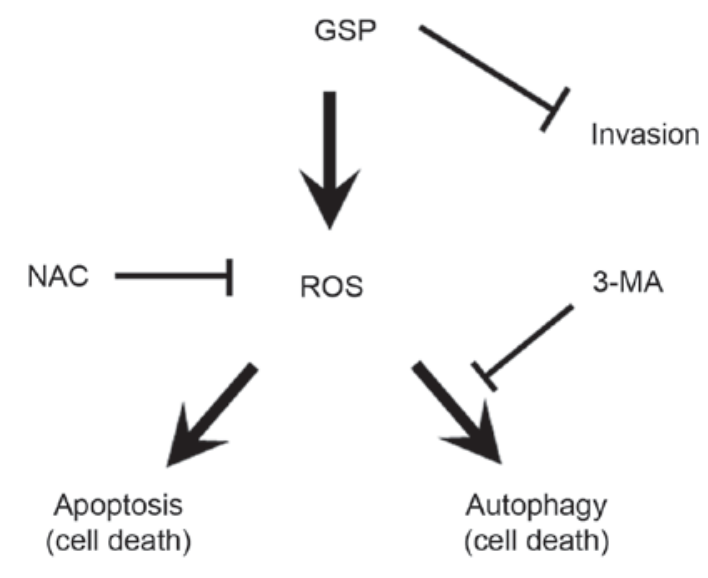

Figure 8. Diagram illustrating the action of GSP in regulating invasion, apoptosis and autophagy in SCC12 cells. GSP inhibits invasion of SCC12 cells by reducing matrix metalloproteinase- 2 expression and activity. GSP also induces apoptosis and autophagic cell death in SCC12 cells, accompanied by increased intracellular ROS production, but the exact mechanism by which this is brought about has to be investigated. GSP, grape seed procyanidins; ROS, reactive oxygen species; 3-MA, 3-methyladenine.

In certain situations, apoptosis and autophagy can occur simultaneously in cells, such that their regulation can be coordinated and the same proteins are involved in both processes. One possible mechanism for simultaneous induction of both apoptosis and autophagy is the stimulation of ROS production. ROS have been shown to regulate apoptosis and autophagy $(30,31)$. The results of the present study suggested that GSP-induced ROS generation caused apoptosis in SCC12 cells, as confirmed by the progression of GSP-treated cells through the sub-G1 phase when also pretreated with NAC. However, pre-treatment of SCC12 cells with 3-MA, an autophagy inhibitor, increased the cell viability, suggesting the presence of an additional mechanism for cell death. The present study hypothesized that GSP-generated ROS also regulated autophagy. Accumulation of LC3-II and GFP-LC3 puncta in SCC12 cells treated with GSP was also observed. However, this autophagic process was diminished by NAC pretreatment of SCC12 cells. Autophagy is a well-established process that controls cell survival and death (11). Autophagy generally stimulates cell survival by sequestering and removing damaged proteins or organelles from cells under conditions of physiological stress (16). The degradation of proteins and/or organelles by autophagy provides essential nutrients necessary for cell survival under certain extreme stress conditions such as starvation or hypoxia. However, cell death may be triggered by the persistent autophagic degradation of proteins or organelles (16). 
In conclusion, GSP inhibited the growth and invasion of SCC12 human cells, and induced ROS-mediated apoptotic and autophagic cell death. These findings provide insights into the association between apoptosis and autophagy induced by GSP, and suggest that regulation of ROS generation and autophagy may be a potential treatment option for SCC.

\section{Acknowledgements}

This study was supported by the Basic Science Research Program through the National Research Foundation of Korea funded by the Ministry of Education (grant no. NRF-2014R1A2A2A01005483).

\section{References}

1. Hassan HM: Protective effects of red grape seed extracts on DNA, brain and erythrocytes against oxidative damage. Glob J Pharmacol 7: 241-248, 2013.

2. Fine AM: Oligomeric proanthocyanidin complexes: History, structure, and phytopharmaceutical applications. Altern Med Rev 5: 144-151,2000.

3. Ariga T: The antioxidative function, preventive action on disease and utilization of proanthocyanidins. Biofactors 21: 197-201, 2004.

4. Jang JK and Han JY: The antioxidant ability of grape seed extracts. Korean J Food Sci Technol 34: 524-528, 2002.

5. Pataki T, Bak I, Kovacs P, Bagchi D, Das DK and Tosaki A: Grape seed proanthocyanidins improved cardiac recovery during reperfusion after ischemia in isolated rat hearts. Am J Clin Nutr 75: 894-899, 2002.

6. Li WG, Zhang XY, Wu YJ and Tian X: Anti-inflammatory effect and mechanism of proanthocyanidins from grape seeds. Acta Pharmacol Sin 22: 1117-1120, 2001.

7. Eng ET, Ye J, Williams D, Phung S, Moore RE, Young MK, Gruntmanis U, Braunstein G and Chen S: Suppression of estrogen biosynthesis by procyanidin dimers in red wine and grape seeds Cancer Res 63: 8516-8522, 2003.

8. Tyagi A, Agarwal R and Agarwal C: Grape seed extract inhibits EGF-induced and constitutively active mitogenic signaling but activates JNK in human prostate carcinoma DU145 cells: Possible role in antiproliferation and apoptosis. Oncogene 22: 1302-1316, 2003.

9. Meeran SM and Katiyar SK: Grape seed proanthocyanidins promote apoptosis in human epidermoid carcinoma A431 cells through alterations in Cdki-Cdk-cyclin cascade, and caspase-3 activation via loss of mitochondrial membrane potential. Exp Dermatol 16: 405-415, 2007.

10. Kaur M, Singh RP, Gu M, Agarwal R and Agarwal C: Grape seed extract inhibits in vitro and in vivo growth of human colorectal carcinoma cells. Clin Cancer Res 12: 6194-6202, 2006.

11. Baehrecke EH: Autophagy: Dual roles in life and death? Nat Rev Mol Cell Biol 6: 505-510, 2005.

12. Shintani T and Klionsky DJ: Autophagy in health and disease: A double-edged sword. Science 306: 990-995, 2004.

13. Kroemer G, Galluzzi L, Vandenabeele P, Abrams J, Alnemri ES, Baehrecke EH, Blagosklonny MV, El-Deiry WS, Golstein P, Green DR, et al: Classification of cell death: Recommendations of the nomenclature committee on cell death 2009. Cell Death Differ 16: 3-11, 2009.
14. Kuma A, Hatano M, Matsui M, Yamamoto A, Nakaya H, Yoshimori T, Ohsumi Y, Tokuhisa T and Mizushima N: The role of autophagy during the early neonatal starvation period. Nature 432: 1032-1036, 2004.

15. Mills KR, Reginato M, Debnath J, Queenan B and Brugge JS: Tumor necrosis factor-related apoptosis-inducing ligand (TRAIL) is required for induction of autophagy during lumen formation in vitro. Proc Natl Acad Sci USA 101: 3438-3443, 2004

16. Lemasters JJ, Qian T, He L, Kim JS, Elmore SP, Cascio WE and Brenner DA: Role of mitochondrial inner membrane permeabilization in necrotic cell death, apoptosis, and autophagy. Antioxid Redox Signal 4: 769-781, 2002.

17. Keston AS and Brandt R: The fluorometric analysis of ultramicro quantities of hydrogen peroxide. Anal Biochem 11: 1-5, 1965.

18. Mantena SK, Baliga MS and Katiyar SK: Grape seed proanthocyanidins induce apoptosis and inhibit metastasis of highly metastatic breast carcinoma cells. Carcinogenesis 27: 1682-1691, 2006.

19. Kabeya Y, Mizushima N, Ueno T, Yamamoto A, Kirisako T, Noda T, Kominami E, Ohsumi Y and Yoshimori T: LC3, a mammalian homologue of yeast Apg8p, is localized in autophagosome membranes after processing. EMBO J 19: 5720-5728, 2000.

20. Mizushima N, Yoshimori T and Levine B: Methods in mammalian autophagy research. Cell 140: 313-326, 2010.

21. Schwartz GK and Shah MA: Targeting the cell cycle: A new approach to cancer therapy. J Clin Oncol 23: 9408-9421, 2005.

22. Lakin ND and Jackson SP: Regulation of p53 in response to DNA damage. Oncogene 18: 7644-7655, 1999.

23. Choi HJ, Lim DY and Park JH: Induction of G1 and G2/M cell cycle arrests by the dietary compound 3,3'-diindolylmethane in HT-29 human colon cancer cells. BMC Gastroenterol 9: 39, 2009.

24. Fulda S and Debatin KM: Sensitization for anticancer drug-induced apoptosis by the chemopreventive agent resveratrol. Oncogene 23: 6702-6711, 2004.

25. Cao Y and Klionsky DJ: Physiological functions of Atg6/Beclin 1: A unique autophagy-related protein. Cell Res 17: 839-849, 2007.

26. Chang KH, Yan MD, Yao CJ, Lin PC and Lai GM: Honokiol-induced apoptosis and autophagy in glioblastoma multiforme cells. Oncol Lett 6: 1435-1438, 2013.

27. Liu B, Cheng Y, Zhang B, Bian HJ and Bao JK: Polygonatum cyrtonema lectin induces apoptosis and autophagy in human melanoma A375 cells through a mitochondria-mediated ROS-p38-p53 pathway. Cancer Lett 275: 54-60, 2009.

28. Ooi KL, Muhammad TS and Sulaiman SF: Growth arrest and induction of apoptotic and non-apoptotic programmed cell death by, Physalis minima L. chloroform extract in human ovarian carcinoma Caov-3 cells. J Ethnopharmacol 128: 92-99, 2010.

29. Sharma SD, Meeran SM and Katiyar SK: Dietary grape seed proanthocyanidins inhibit UVB-induced oxidative stress and activation of mitogen-activated protein kinases and nuclear factor-kappaB signaling in in vivo SKH-1 hairless mice. Mol Cancer Ther 6: 995-1005, 2007.

30. Scherz-Shouval R, Shvets E, Fass E, Shorer H, Gil L and Elazar Z: Reactive oxygen species are essential for autophagy and specifically regulate the activity of Atg4. EMBO J 26: 1749-1760, 2007.

31. Gao M, Yeh PY, Lu YS, Hsu CH, Chen KF, Lee WC, Feng WC, Chen CS, Kuo ML and Cheng AL: OSU-03012, a novel celecoxib derivative, induces reactive oxygen species-related autophagy in hepatocellular carcinoma. Cancer Res 68: 9348-9357, 2008. 